\title{
$K$-ras gene mutation as a predictor of cancer cell responsiveness to metformin
}

\author{
YU MA, FU-CHUN GUO, WEI WANG, HUA-SHAN SHI, DAN LI and YONG-SHENG WANG \\ Department of Thoracic Oncology, Cancer Center, State Key Laboratory of Biotherapy, \\ West China Hospital, Sichuan University, Chengdu, Sichuan 610041, P.R. China
}

Received March 11, 2013; Accepted July 3, 2013

DOI: $10.3892 / \mathrm{mmr} .2013 .1596$

\begin{abstract}
An increasing number of studies support the use of metformin, a common antidiabetic drug, as a novel anticancer therapeutic. However, its mechanism of action has yet to be identified. In the current study, metformin was observed to effectively inhibit the growth of the K-ras mutant but not wild-type tumors in vivo. The antitumor effects of metformin were mediated by the induction of apoptosis and inhibition of proliferation in vivo. In addition, metformin induced apoptosis in the K-ras mutant tumors, A549 and PANC-1, but not in the K-ras wild-type tumor, A431, in vitro. Similarly, at lower concentrations, metformin inhibited cell proliferation in the K-ras mutant, but not in the K-ras wild-type tumor cells in vitro. These observations indicate that tumors with K-ras mutations are sensitive to metformin therapy. In addition, metformin significantly arrested K-ras mutant and wild-type tumor cells in G1 phase in vitro and metformin downregulated two important downstream effectors of the Ras signaling pathway in K-ras mutant tumors. Metformin was concluded to function as a potential K-ras-targeting agent that has potential for cancer therapy.
\end{abstract}

\section{Introduction}

Previous epidemiological studies have hypothesized an association between diabetes and cancer. Firstly, the incidence of several types of cancer, including pancreatic, liver, breast, colorectal, urinary tract and of the female reproductive organs, is increased in diabetic patients (1). Secondly, cancer patients with diabetes have been reported to respond poorly to cancer chemotherapy and have a poorer prognosis than non-diabetic patients (2).

Correspondence to: Dr Yong-Sheng Wang, Department of Thoracic Oncology, Cancer Center, State Key Laboratory of Biotherapy, West China Hospital, Sichuan University, No. 1 Keyuan Road, 4th Gaopeng Avenue, Chengdu, Sichuan 610041, P.R. China E-mail: wangys@scu.edu.cn

Key words: K-ras, molecular targeting, non-small cell lung cancer, pancreatic cancer, epidermoid cancer, metformin
Previous studies investigating patients with type 2 diabetes have reported that metformin significantly reduces cancer incidence and improves the survival of these cancer patients (3). Metformin is the most widely used antidiabetic drug, functioning to reduce circulating glucose levels, increase insulin sensitivity and reduce insulin resistance-associated hyperinsulinemia (4). A number of studies have reported that metformin is a novel anticancer drug that may be used to treat specific types of cancer, including breast, ovarian, endometrial and pancreatic (5-8). However, Bodmer et al reported that metformin did not decrease the risk of lung cancer and colon cancer $(9,10)$. This may be ascribed to the various genetic backgrounds of the numerous tumor types analyzed. For example, Buzzai et al reported that metformin selectively impairs p53-deficient tumor cell growth (11). To date, there has been extensive epidemiological and retrospective studies that support the antineoplastic effects of metformin (4). However, the precise mechanisms by which metformin exerts its anti-tumor effects remain unclear.

Ras proteins are prototypical G-proteins that have been shown to play key roles in signal transduction, proliferation and differentiation (12). Under normal conditions, Ras is activated transiently in a stimulus-dependent fashion. By contrast, mutated Ras proteins are constitutively active and thus, persistently stimulate growth or differentiation (13). Therefore, mutationally activated Ras genes are hypothesized to represent important targets for cancer therapy. K-, H-and N-Ras of the Ras family are central in regulating diverse cellular functions important for growth, differentiation and survival. Of the Ras proteins, K-ras is the most frequently mutated (14). A number of strategies have been developed to target K-ras and specific agents have been demonstrated to effectively treat cancer (15). However, agents that specifically target K-ras with no toxicity in normal cells have not yet been developed (16).

A previous study found that insulin induces activation of the Ras gene (4). In addition, other studies have reported that metformin reduces the levels of circulating insulin $(17-19,20)$. Thus, we hypothesized that metformin exerts its antitumor effects by reducing activation of the Ras gene. In the current study, metformin was found to effectively inhibit the growth of K-ras-mutated tumors but not K-ras wild-type tumors. In addition, the antitumor effects of metformin were found to be mediated via induction of apoptosis and inhibition of proliferation. Downregulation of K-ras downstream signaling pathways 
contributed to the inhibition of proliferation and induction of apoptosis. In conclusion, observations of the current study hypothesize the use of metformin to inhibit the growth of tumors with various molecular signatures and in particular with different K-ras backgrounds.

\section{Materials and methods}

Cells and reagents. K-ras mutant tumor cells [human A549 lung adenocarcinoma (21) and human PANC-1 pancreatic (22)] and K-ras wild-type tumor cells [human A431 epidermoid adenocarcinoma (23)] were purchased from American Type Culture Collection (Manassas, VA, USA) and were cultured according to the manufacturer's instructions. Metformin was purchased from Sigma-Aldrich (St. Louis, MO, USA) and epidermal growth factor (EGF) was obtained from Shanghai PrimeGene Bio-tech Co., Ltd., (Shanghai, China).

Tumor growth assay. Seven-week-old female athymic (BALB/c, nu/nu) mice used for the in vivo NSCLC xenograft study were purchased from Beijing HFK Bioscience Co., Ltd. (Beijing, China). A549 $\left(10^{7}\right)$, PANC-1 $\left(10^{7}\right)$ and A431 cells $\left(10^{7}\right)$ were injected subcutaneously into the flanks of the female nude mice and allowed to grow to a density of $100 \mathrm{~mm}^{3}$. Animals were randomly divided into two groups of six mice. Control mice received daily intragastric administration of the vehicle solution for metformin, $\mathrm{H}_{2} \mathrm{O}$, while treated mice received daily intragastric administration of metformin $(250 \mathrm{mg} / \mathrm{kg})$.

Detection of Ki67 expression within tumors. Tumor tissue was removed from tumor-bearing nude mice following the final treatment and immunohistochemical analysis was performed with an anti-Ki67 antibody (1:100; Thermo Fisher Scientific Inc., Waltham, MA, USA) to determine the expression of Ki67.

Terminal deoxynucleotidyl transferase-mediated dUTP nick-end labeling (TUNEL) assay in tumors in situ. Tumor tissue was removed from tumor-bearing nude mice following the final treatment and apoptosis levels in vivo were determined using a TUNEL assay according to the manufacturer's instructions (Promega Corporation, Madison, WI, USA).

Lactic acid assay. A549, PANC-1 and A431 cells were seeded at $2.5 \times 10^{5}$ cells/well in 6 -well plates in the appropriate media overnight and treated with metformin at four concentrations $(0,1,5$ and $10 \mathrm{mM})$ for an additional $48 \mathrm{~h}$. Supernatants of the cell culture media were harvested and the concentration of lactic acid was analyzed using a lactic acid assay according to the manufacturer's instructions (Nanjing Jiancheng Bioengineering Institute, Nanjing, China). Viable cells were counted and the lactic acid release/cell was calculated as follows: concentrations of lactic acid/cell densities.

Cell cycle analysis by flow cytometry. A549, PANC-1 and A431 cells were seeded at $2.5 \times 10^{5}$ cells/well in 6-well plates in the appropriate media overnight. Subsequently, cells were starved for $24 \mathrm{~h}$ and treated with $10 \%$ serum and four concentrations of metformin for $24 \mathrm{~h}$. Cells were harvested, washed twice with PBS, fixed in a $70 \%$ methanol solution and stored at $4^{\circ} \mathrm{C}$ overnight. Following fixation, cells were resuspended and stained with PI and analyzed using flow cytometry (Beckman Coulter Inc., Brea, CA, USA).

Methylthiazolyldiphenyl-tetrazolium bromide (MTT) cell proliferation assay. A549, PANC-1 and A431 cells were treated with varying doses of metformin $(0,0.5,1,2.5,5$ and $10 \mathrm{mM})$ for $72 \mathrm{~h}$. MTT (5 mg/ml; Sigma-Aldrich) was added to the 96-well plates at $10 \mu \mathrm{l} /$ well and the plates were incubated for an additional hour. The MTT reaction was terminated by the addition of $100 \mu 1$ DMSO. Subsequently, MTT assay results were obtained by measuring the absorption of each well at $490 \mathrm{~nm}$.

Apoptosis analysis by flow cytometry. A549, PANC-1 and A431 cells were seeded at $2.5 \times 10^{5}$ cells/well in 6-well plates in the appropriate media overnight and were treated with $10 \%$ serum and four concentrations of metformin for an additional $24 \mathrm{~h}$. Cells were harvested, washed twice with PBS, resuspended and stained with PI and apoptosis levels were analyzed using flow cytometry (Beckman Coulter, Inc.).

Western blot analysis. A549, PANC-1 and A431 cells were seeded at $2 \times 10^{5}$ cells/well in 6-well plates in the appropriate media and treated with two concentrations of metformin (0 and $5 \mathrm{mM}$ ) for $24 \mathrm{~h}$. Cell lysates were prepared and analyzed by western blot analysis as previously described (7). Antibodies against phospho-T172 adenosine monophosphate activated protein kinase (AMPK), mitogen-activated protein kinase (MAPK), phospho-T202/204 MAPK, AKT (protein kinase B), phospho-T308 AKT and p53 were purchased from Cell Signaling Technology, Inc. (Danvers, MA, USA). The antibody against AMPK was purchased from Santa Cruz Biotechnology, Inc. (Santa Cruz, CA, USA). Peroxidase-labeled antibodies against mouse and rabbit were used as secondary antibodies (Beijing Zhongshan Golden Bridge Biotechnology Co., Ltd., Beijing, China).

Statistical analysis. Data are presented as the mean \pm SEM from a minimum of three independent experiments, unless otherwise noted. Statistical analyses were performed by Student's t-test. $\mathrm{P}<0.05$ was considered to indicate a statistically significant difference.

\section{Results}

Differential inhibition of xenograft tumor growth induced by metformin on various K-ras backgrounds. To gain an improved understanding of the effects of metformin treatment on cancer cell xenografts in vivo, mice were administered metformin orally. As shown in Fig. 1, treatment with metformin resulted in significant inhibition of human A549 and PANC-1 (both $\mathrm{P}<0.05$ ) but not A431 xenograft tumor growth $(\mathrm{P}>0.05)$.

Metformin induces apoptosis and inhibits the proliferation of $K$-ras-mutated tumor cells in vivo. The effects of metformin on cell proliferation in vivo were determined by staining sections from each group with an anti-Ki67 antibody. As presented in Fig. 2A, treatment with metformin resulted in significant inhibition of cell proliferation in A549 and PANC-1 
A

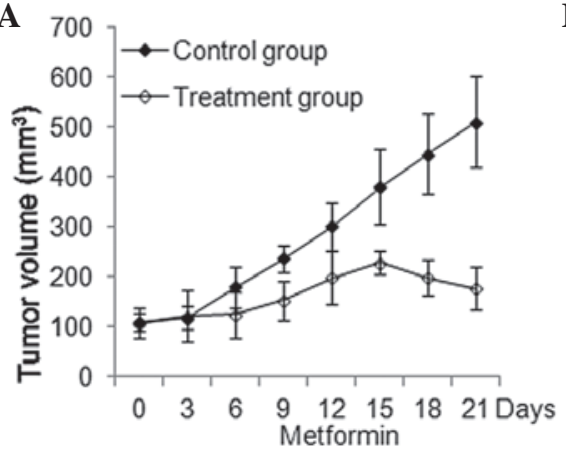

B

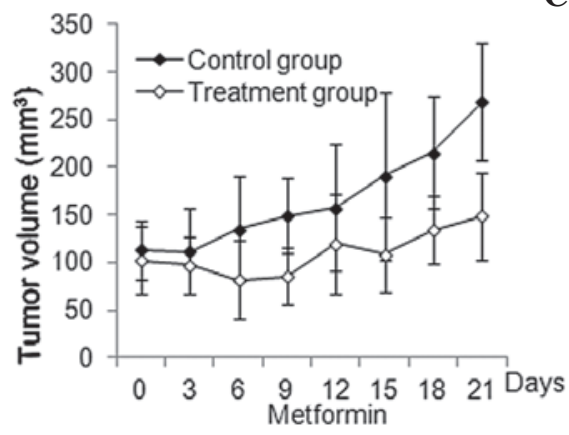

C

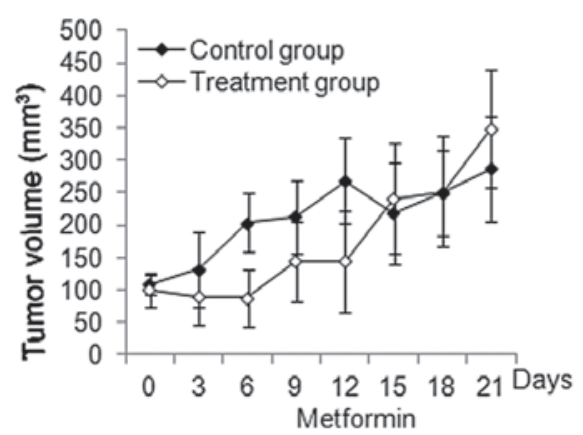

Figure 1. Effects of metformin on cancer cell xenografts in vivo. (A and B) Daily intragastric administration of metformin ( $250 \mathrm{mg} / \mathrm{kg}$ ) resulted in significantly increased regression of established A549 and PANC-1 cell xenograft tumors (both $\mathrm{P}<0.05$ ). (C) Increased regression was not observed in A431 cell xenograft tumors $(\mathrm{P}>0.05)$.

A A549 PANC-1 A431

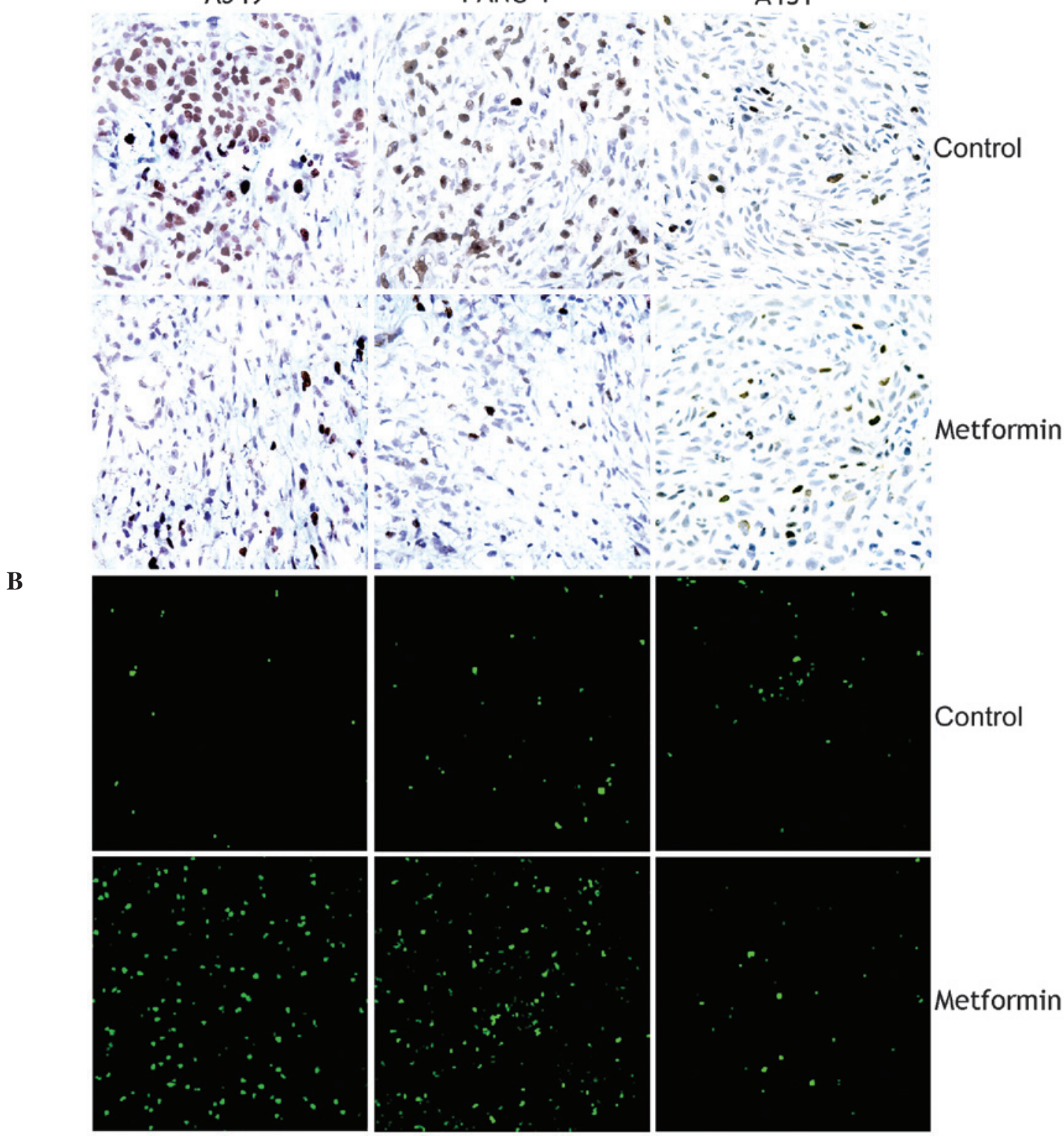

Figure 2. Effects of metformin on proliferation and apoptosis in vivo. (A) Metformin inhibited the proliferation of cancer cells in vivo. Proliferating tumor cells were detected using an anti-Ki67 antibody and positive cell densities were quantified by counting the number of cells/high power field (magnification, $\mathrm{x} 400$ ). There was a significant difference observed in the number of proliferating tumor cells between treated and control groups of A549 and PANC-1 cells (P<0.05) but not A431 cells in vivo ( $\mathrm{P}>0.05)$. (B) Metformin induced apoptosis of cancer cells in vitro. Detection of apoptotic cancer cells using a TUNEL assay. The percentage of apoptotic cells was determined by counting the number of apoptotic cells divided by the total number of cells in the field (five high power fields/slide; magnification, $\mathrm{x} 400$ ). There was a significant difference in the percentage of apoptotic tumor cells between treated and control groups of A549 and PANC-1 cells $(\mathrm{P}<0.05)$ but not A431 cells in vivo $(\mathrm{P}>0.05)$. TUNEL, terminal deoxynucleotidyl transferase-mediated dUTP nick-end labeling. 
A

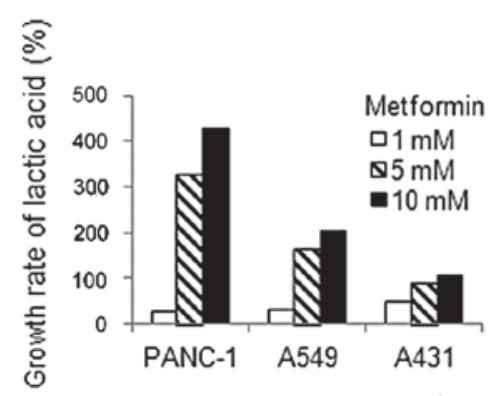

D

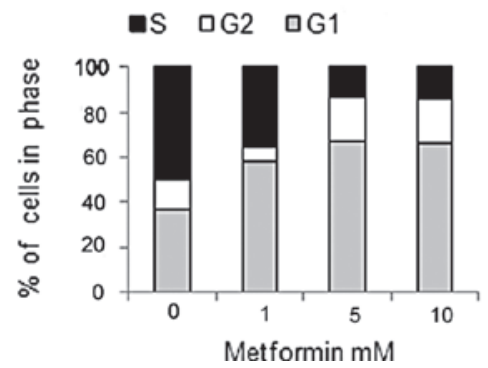

B

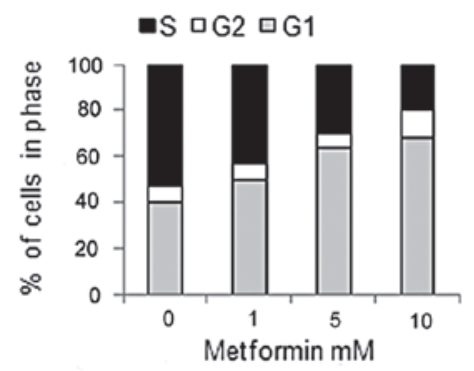

$\mathbf{E}$

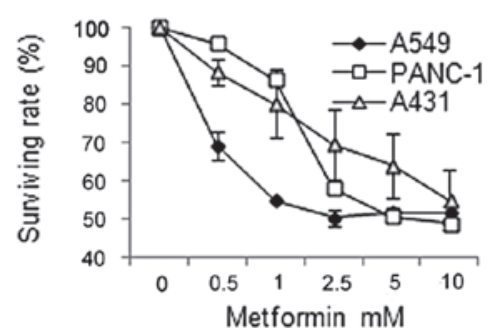

C
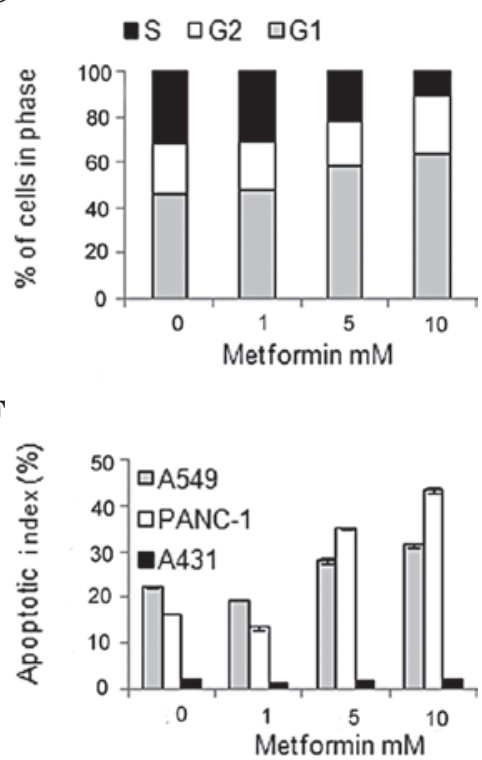

Figure 3. Effects of metformin on glycolysis, cell cycle progression, cell proliferation and apoptosis in vitro. (A) Metformin increased glycolysis in A549, PANC-1 and A431 cells. Lactic acid concentration was determined by a lactic acid assay and the lactic acid release of a single cell was calculated as the concentration of lactic acid/cell density. Metformin inhibited cell cycle progression by arresting the (B) A549, (C) PANC-1 and (D) A431 cells in G1 phase. (E) Cell cycle analysis was performed by flow cytometry. Metformin inhibited the proliferation of cancer cells in a dose-dependent manner. A549, PANC-1 and A431 cells were cultured in the presence of increasing concentrations of metformin for $72 \mathrm{~h}$. The relative growth of the cells was determined by MTT assay. (F) Metformin induced apoptosis in A549 and PANC-1 cells but not in A431 cells and the percentage of apoptotic cells was determined by flow cytometry. MTT, methylthiazolyldiphenyl-tetrazolium bromide.

cell xenografts (both $\mathrm{P}<0.05$ ) but not in A431 cell xenografts $(\mathrm{P}>0.05)$. Next, a TUNEL assay was performed to examine the effects of metformin on apoptosis in vivo. As shown in Fig. 2B, treatment with metformin resulted in significant induction of apoptosis in A549 and PANC-1 cell xenografts (both $\mathrm{P}<0.05$ ) but not in A431 cell xenografts $(\mathrm{P}>0.05)$.

Metformin increases glycolysis and induces cell cycle arrest and apoptosis while inhibiting proliferation ex vivo. To understand the effects of metformin treatment on glycolysis, the concentration of lactic acid in the supernatants of the cell culture media was quantified and the lactic acid release/cell was analyzed following treatment with metformin. As presented in Fig. 3A, treatment with metformin resulted in elevated levels of glycolysis in the three cancer cell lines.

Effects of metformin treatment on cell cycle progression were determined by flow cytometry analysis following treatment with metformin. As presented in Fig. 3B-D, metformin significantly blocked serum-induced entry into $\mathrm{S}$ phase in a dose-dependent manner, resulting in cell cycle arrest in $\mathrm{G} 1$ phase in the three cancer cell lines following $24 \mathrm{~h}$ metformin treatment.

To determine the effects of metformin treatment on cell proliferation, an MTT assay was performed following treatment with metformin (24). As presented in Fig. 3E, metformin inhibited cell growth in a dose-dependent manner in all three cancer cell lines following $72 \mathrm{~h}$ metformin treatment. The mean $\mathrm{IC}_{50}$ value was $\sim 5 \mathrm{mM}$ for A549 and PANC-1 cells and $\sim 10 \mathrm{mM}$ for A431 cells.

The effects of metformin treatment on apoptosis were investigated by flow cytometry analysis following treatment with metformin. As presented in Fig. 3F, metformin induced

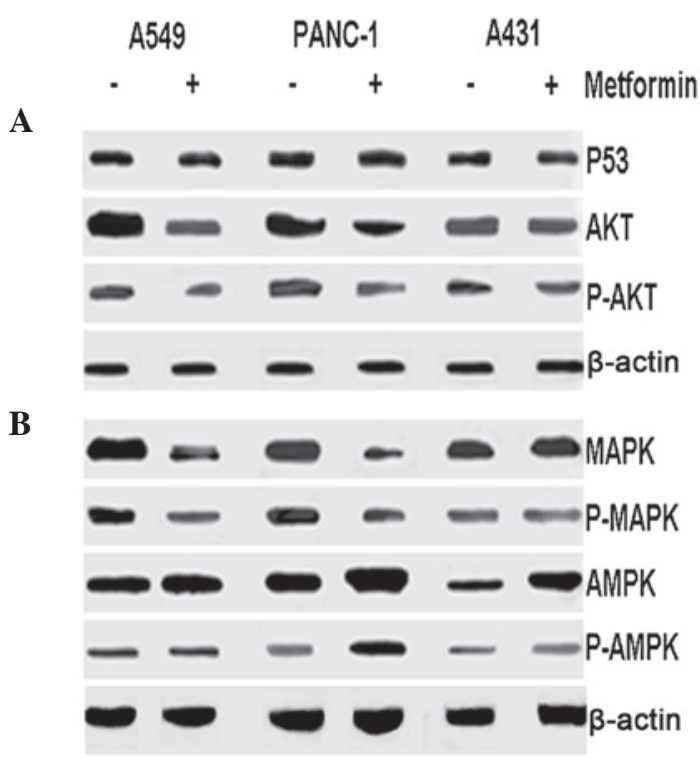

Figure 4. Effects of metformin on cell signaling pathways. (A) Metformin did not affect the protein expression of p53 in A549, PANC-1 and A431 cells. Metformin inhibited AKT downstream of Ras signaling pathways in A549 cells and PANC-1 cells but not in A431 cells. (B) Metformin inhibited MAPK in A549 cells and PANC-1 cells but not in A431 cells. Metformin activated AMPK in PANC-1 cells and A431 cells but not in A549 cells. Untreated control A549 cells, PANC-1 cells and A431 cells pretreated with 5 mM metformin for $24 \mathrm{~h}$ were stimulated with EGF ( $100 \mathrm{ng} / \mathrm{ml})$ for $60 \mathrm{~min}$ before cell lysis. Then, the cell lysates were used for western blot analyses. AMPK. adenosine monophosphate activated protein kinase; MAPK, mitogen-activated protein kinases; AKT, protein kinase B.

apoptosis in A549 and PANC-1 cells. The percentage change ranged between 9.2 and $27.2 \%$, depending on the cell line. However, metformin did not induce apoptosis in A431 cells. 
Metformin inhibits the downstream signaling effectors of $K$-ras. Western blotting was performed on the three cell lines following treatment with metformin to determine its underlying anti-tumor mechanisms. As presented in Fig. 4, expression of p53 was not affected by metformin in A549, PANC-1 and A431 cells. Treatment with metformin resulted in decreases in MAPK, p-MAPK, AKT and p-AKT levels in A549 and PANC-1 cells but not in A431 cells. However, metformin treatment increased AMPK and p-AMPK in PANC-1 cells and A431 cells, but not in A549 cells.

\section{Discussion}

An increasing number of studies have implicated metformin as a potentially effective anticancer drug; however, the mechanism of action remains unclear. Observations of the current study indicate that metformin specifically targets K-ras to elicit its anticancer effects.

Using an in vivo mouse model, metformin was observed to only exert anticancer effects in K-ras mutant tumors, but not in K-ras wild-type tumors. To understand the mechanisms of these effects in vitro, the effects of metformin on glycolysis were investigated. As described by Warburg et al, tumor cells maintain a high glycolytic rate even in conditions of adequate oxygen supply (25). Therefore, interfering with aerobic glycolysis represents a potentially effective strategy to selectively target cancer cells. Metformin increased the rate of glycolysis in K-ras mutant and wild-type cell lines, indicating that the anticancer effects of metformin are mediated through pathways other than aerobic glycolysis.

Next, the effects of metformin on cell cycle progression and proliferation were investigated. Metformin was observed to significantly arrest the cells in G1 phase and inhibit the proliferation of K-ras mutant and wild-type tumors. These results did not highlight an explanation for the differential efficacy of metformin on K-ras mutant and wild-type tumors in vivo. Therefore, the effects of metformin on apoptosis were investigated and it was observed that metformin induces apoptosis in K-ras mutant tumors but not in K-ras wild-type tumors. This indicates that metformin is likely to inhibit tumor growth of K-ras mutant tumors by increasing apoptosis and inhibiting proliferation, which is consistent with the in vivo results. The results indicate that the anticancer activity of metformin is primarily due to the induction of apoptosis and that metformin is cytostatic and cytotoxic to K-ras mutant tumors. To gain a full understanding of the underlying mechanisms by which metformin induces apoptosis, p53 expression, an essential component of apoptosis induction was investigated. Expression of p53 was not affected by metformin treatment in all three cancer cell lines and observations made by Buzzai et al support the hypothesis that p53 is unnecessary for apoptosis induction by metformin (11).

In the current study, metformin was observed to target important downstream effectors of the Ras signaling pathway in cancer therapy, including AKT. Akt/PKB is a downstream target of PI3-K that plays a significant role in the regulation of apoptosis by activating proapoptotic proteins, including Bad and caspase 9 (26). Following metformin treatment, AKT levels were identified to decrease in A549 and PANC-1 cells but not in A431 cells. These observations indicate that metformin induces apoptosis by inhibiting AKT rather than via the activation of p53. A previous study reported that metformin may exert antitumor effects via the downregulation of AKT (27).

An additional important downstream effector of the Ras signaling pathway is MAPK, which mediates the anticancer effects of metformin. MAPK activation results in the transcription of a number of genes associated with proliferation (28). Metformin treatment was observed to reduce MAPK and p-MAPK levels in A549 and PANC-1 cells. However, although metformin did not downregulate MAPK in A431 cells, the proliferation of these cells was inhibited, indicating that alternative mechanisms underlie the anti-proliferative effect of metformin in these cells.

It has been widely reported that metformin may function as an AMPK activator to target the LKB1/AMPK signaling pathway in cancer therapy (29). Thus, the impacts of metformin on AMPK activity in K-ras mutant and wild-type cell lines were determined. AMPK is a cellular energy sensor that inhibits tumorigenesis via the regulation of cell growth, cell proliferation, autophagy, stress responses and cell polarity (30). Treatment with metformin was observed to activate AMPK in PANC-1 and A431 cells but not in A549 cells. This indicates that activation of AMPK is a key mediator of the anti-proliferative mechanisms of metformin in PANC-1 and A431 cells. Therefore, the current study demonstrates an inhibition of proliferation by metformin via the downregulation of MAPK or the activation of AMPK. Once the LKBI gene is mutated, metformin may only inhibit proliferation by downregulating MAPK. These observations are consistent with previous studies (31-33).

In summary, the present study indicates that metformin targets K-ras and may therefore represent a drug target for the treatment of cancer. Mutational activation of Ras genes is associated with $33 \%$ of human cancers, hence, Ras is considered to be an important target for cancer therapy (34). However, an ideal agent that specifically targets K-ras has not yet been developed $(14,16)$. Metformin is orally active, with a relatively favorable toxicity profile and is low in cost. The promising results of our preclinical study are consistent with previous studies analyzing metformin treatment in clinical settings (35). Since the current observations are preliminary, in depth molecular profiling of the patient's tumor must be performed prior to cancer treatment with metformin.

\section{Acknowledgements}

This study was supported by a grant from the National Major Project (no. 2011ZX09302-001-01).

\section{References}

1. Vigneri P,Frasca F, Sciacca L, Pandini G and Vigneri R: Diabetes and cancer. Endocr Relat Cancer 16: 1103-1123, 2009.

2. Gallagher EJ and LeRoith D: Insulin, insulin resistance, obesity, and cancer. Curr Diab Rep 10: 93-100, 2010.

3. Martin-Castillo B, Vazquez-Martin A, Oliveras-Ferraros C and Menendez JA: Metformin and cancer: doses, mechanisms and the dandelion and hormetic phenomena. Cell Cycle 9: 1057-1064, 2010.

4. Gonzalez-Angulo AM and Meric-Bernstam F: Metformin: a therapeutic opportunity in breast cancer. Clin Cancer Res 16: $1695-1700,2010$ 
5. Zakikhani M, Dowling R, Fantus IG, Sonenberg N and Pollak M: Metformin is an AMP kinase-dependent growth inhibitor for breast cancer cells. Cancer Res 66: 10269-10273, 2006.

6. Jones RG, Plas DR, Kubek S, et al: AMP-Activated protein kinase induces a p53-dependent metabolic checkpoint. Mol Cell 18: 283-293, 2005.

7. Cantrell LA, Zhou C, Mendivil A, Malloy KM, Gehrig PA and Bae-Jump VL: Metformin is a potent inhibitor of endometrial cancer cell proliferation - implications for a novel treatment strategy. Gynecol Oncol 116: 92-98, 2010.

8. Kisfalvi K, Sinnett-Smith J, Eibl G and Rozengurt E: Metformin inhibits growth of human pancreatic cancer cells in vitro and in vivo. Pancreas 38: 1016-1017, 2009.

9. Bodmer M, Becker C, Jick SS and Meier CR: Metformin does not alter the risk of lung cancer: a case-control analysis. Lung Cancer 78: 133-137, 2012.

10. Bodmer M, Becker C, Meier C, Jick SS and Meier CR: Use of metformin is not associated with a decreased risk of colorectal cancer: a case-control analysis. Cancer Epidemiol Biomarkers Prev 21: 280-286, 2012.

11. Buzzai M, Jones RG, Amaravadi RK, et al: Systemic treatment with the antidiabetic drug metformin selectively impairs p53-deficient tumor cell growth. Cancer Res 67: 6745-6752, 2007.

12. Reuter CW, Morgan MA and Bergmann L: Targeting the Ras signaling pathway: a rational, mechanism-based treatment for hematologic malignancies? Blood 96: 1655-1669, 2000.

13. Cox AD and Der CJ: Ras family signaling: therapeutic targeting. Cancer Biol Ther 1: 599-606, 2002.

14. Friday BB and Adjei AA: K-ras as a target for cancer therapy. Biochim Biophys Acta 1756: 127-144, 2005.

15. Legaspi A, Jeevanandam M, Starnes HF Jr and Brennan MF: Whole body lipid and energy metabolism in the cancer patient. Metabolism 36: 958-963, 1987.

16. Blum R and Kloog Y: Tailoring Ras-pathway - inhibitor combinations for cancer therapy. Drug Resist Updat 8: 369-380, 2005.

17. Skolnik EY, Batzer A, Li N, et al: The function of GRB2 in linking the insulin receptor to Ras signaling pathways. Science 260 : 1953-1955, 1993

18. Noguchi T, Matozaki T, Horita K, Fujioka Y and Kasuga M: Role of SH-PTP2, a protein-tyrosine phosphatase with Src homology 2 domains, in insulin-stimulated Ras activation. Mol Cell Biol 14: 6674-6682, 1994.

19. Desbois-Mouthon C, Cadoret A, Blivet-Van Eggelpoël MJ, et al: Insulin and IGF-1 stimulate the beta-catenin pathway through two signalling cascades involving GSK-3beta inhibition and Ras activation. Oncogene 20: 252-259, 2001.
20. Medema RH, de Vries-Smits AM, van der Zon GC, Maassen JA and Bos JL: Ras activation by insulin and epidermal growth factor through enhanced exchange of guanine nucleotides on p21ras. Mol Cell Biol 13: 155-162, 1993.

21. Lehman TA, Bennett WP, Metcalf RA, et al: p53 mutations, ras mutations and p53-heat shock 70 protein complexes in human lung carcinoma cell lines. Cancer Res 51: 4090-4096, 1991.

22. Watanabe M, Nobuta A, Tanaka J and Asaka M: An effect of K-ras gene mutation on epidermal growth factor receptor signal transduction in PANC-1 pancreatic carcinoma cells. Int J Cancer 67: 264-268, 1996.

23. Oliveras-Ferraros C, Cufi S, Queralt B, et al: Cross-suppression of EGFR ligands amphiregulin and epiregulin and de-repression of FGFR3 signalling contribute to cetuximab resistance in wild-type KRAS tumour cells. Br J Cancer 106: 1406-1414, 2012.

24. Iivanainen E, Lauttia S,Zhang N, et al: The EGFR inhibitor gefitinib suppresses recruitment of pericytes and bone marrow-derived perivascular cells into tumor vessels. Microvasc Res 78: 278-285, 2009.

25. Bartrons R and Caro J: Hypoxia, glucose metabolism and the Warburg's effect. J Bioenerg Biomembr 39: 223-229, 2007.

26. Feig LA and Buchsbaum RJ: Cell signaling: life or death decisions of ras proteins. Curr Biol 12: R259-R261, 2002

27. Ferla R, Haspinger E and Surmacz E: Metformin inhibits leptin-induced growth and migration of glioblastoma cells. Oncol Lett 4: 1077-1081, 2012.

28. Adjei AA: Blocking oncogenic Ras signaling for cancer therapy. J Natl Cancer Inst 93: 1062-1074, 2001.

29. Wang W and Guan KL: AMP-activated protein kinase and cancer. Acta Physiol (Oxf) 196: 55-63, 2009.

30. Carling D: AMP-activated protein kinase: balancing the scales. Biochimie 87: 87-91, 2005.

31. Mahoney CL, Choudhury B, Davies H, et al: LKB1/KRAS mutant lung cancers constitute a genetic subset of NSCLC with increased sensitivity to MAPK and mTOR signalling inhibition. Br J Cancer 100: 370-375, 2009.

32. Ben Sahra I, Regazzetti C, Robert G, et al: Metformin, independent of AMPK, induces mTOR inhibition and cell-cycle arrest through REDD1. Cancer Res 71: 4366-4372, 2011.

33. Kalender A, Selvaraj A, Kim SY, et al: Metformin, independent of AMPK, inhibits mTORC1 in a rag GTPase-dependent manner. Cell Metab 11: 390-401, 2010.

34. Cox AD and Der CJ: Farnesyltransferase inhibitors: promises and realities. Curr Opin Pharmacol 2: 388-393, 2002.

35. Li D: Metformin as an antitumor agent in cancer prevention and treatment. J Diabetes 3: 320-327, 2011. 